\title{
“HUNSRÜCKISCHE” MERKMALE IN DER UMGANGSSPRACHE DER COLONIAS UNIDAS
}

\author{
Rasgos del dialecto "Hunsrückisch" en el alemán hablado en Colonias Unidas
}

Anita Lotholz ${ }^{1}$

Recibido 29/11/2019

Aceptado 29/01/2020

\begin{abstract}
Die Umgangssprache der Colonias Unidas hatte schon seit längerem die Aufmerksamkeit der Autorin auf sich gezogen. Die sprachlichen Merkmale, die nicht mit dem Hochdeutsch übereinstimmten und die hunsrückischen Lexeme, die ihr unbekannt waren, führten zu dem Wunsch diese Sprache näher zu erforschen. Die größte Schwierigkeit der Forschungsarbeit war die Beschränktheit an Referenzmaterial zu den Merkmalen des Hunsrückisch. Es ist wichtig klarzustellen, dass in dieser Arbeit Pionierarbeit geleistet wird, da in Paraguay noch keine Forschung dieser Art zu dem Hunsrückisch durchgeführt wurde. Das Hauptziel der vorliegenden Arbeit betrifft die Merkmale des Hunsrückisch in den Colonias Unidas. Anhand von Gesprächen mit Informanten sollten Gemeinsamkeiten und/oder Unterschiede mit dem Hunsrückisch in Rio Grande do Sul festgestellt und analysiert werden. Das empirische Material wurde anhand von Interviews mit älteren Bewohnern der Colonias Unidas erhalten, und anschließend mit phonologischen, morphologischen, syntaktischen und lexikalischen Merkmalen des Hunsrückisch in Brasilien verglichen. Die Ergebnisse dieser Forschung sind signifikant in Hinsicht darauf, dass das Auftreten verschiedener Merkmale des Hunsrückisch in der Umgangssprache der Colonias Unidas bewiesen wird. Dieses Ergebnis ist für die Autorin persönlich zufriedenstellend, da sie die Fragestellung über die Anwesenheit des Hunsrückisch in Paraguay beantworten.
\end{abstract}

Schlüsselwörter: Hunsrückisch, Lingüistik, Paraguay

\section{RESUMEN}

El lenguaje coloquial de las Colonias Unidas ya había llamado tiempo atrás la atención de la autora. Las características lingüísticas, que no concuerdan con el alemán estándar y los lexemas del Hunsrückisch, desconocidos para ella, le llevaron a querer investigar más sobre este idioma. La mayor dificultad de este trabajo fue el poco material disponible sobre las características del Hunsrückisch. Es importante aclarar que este trabajo es innovador, ya que en Paraguay aún no existe investigación de este tipo sobre el Hunsrückisch. El objetivo principal del presente artículo apunta a establecer las características del Hunsrückisch en las Colonias Unidas. En base a entrevistas con informantes se deberán constatar y analizar características en común y/o diferencias con el Hunsrückisch de Rio Grande do Sul. El material empírico fue obtenido mediante entrevistas con pobladores mayores de las Colonias Unidas y seguidamente comparado con las características fonológicas, morfológicas, sintácticas y léxicas del Hunsrückisch en Brasil. Los resultados de esta investigación son significantes en el sentido que es comprobada la existencia de varias características del Hunsrückisch en el lenguaje coloquial de las Colonias Unidas. Este resultado es satisfactorio para la autora, ya que se obtiene una respuesta a la pregunta sobre la existencia del Hunsrückisch en Paraguay.

Palabras claves: Hunsrückisch, Lingüística, Paraguay

\section{ABSTRACT}

The colloquial language of the United Colonies had long ago drawn the author's attention. The linguistic characteristics, which do not agree with standard German and the Hunsrückisch lexemes, unknown to her, led her to want to investigate more about this language. The greatest difficulty of this work was the lack of material

\footnotetext{
${ }^{1}$ Instituto Superior de Lenguas, Facultad de Filosofía, Universidad Nacional de Asunción. anita.lotholz@gmail.com
} 
available on the characteristics of the Hunsrückisch in Paraguay. It is important to clarify that this work is innovative, since in Paraguay there is still no research of this type on the Hunsrückisch. The main objective of this article aims to establish the characteristics of the Hunsrückisch dialect spoken in the United Colonies. Based on interviews with informants, common characteristics and/or differences with the Hunsrückisch dialect of Rio Grande do Sul were verified and analyzed. The empirical material was obtained through interviews with older residents of the United Colonies and then compared with the phonological, morphological, syntactic and lexical characteristics, of the Hunsrückisch dialect in Brazil. The results of this investigation are significant in the sense that the existence of several characteristics of the Hunsrückisch dialect in the colloquial language of the United Colonies in Paraguay is verified. This result is significant, since it provided an answer to the question about the existence of the Hunsrückisch in Paraguay.

Keywords: Hunsrückisch, Linguistics, Paraguay

Eine Sprachforschung betrifft nicht nur die Sprache einer Gesellschaft, sondern auch die Gesellschaft selbst. Man kann die Sprache nicht von der Gesellschaft trennen, da sie zusammengehören. Die Sprache verändert sich durch Völkerwanderungen und durch den Kontakt, den die Menschen zu anderen knüpfen. Sie nimmt neue Merkmale an und verwirft andere im Laufe der Zeit.

Deutschland ist ein Land, in dem es außer Hochdeutsch oder Standarddeutsch, wie wir es in dieser Arbeit benennen werden, viele Dialekte gibt. Dazu kann man auch das Hunsrückisch zählen. Das Hunsrückisch ist ein Dialekt, das in Deutschland in der Gegend zwischen dem Rhein und der Moselfränkischen Region gesprochen wird und das durch die Migration einer bestimmten Völkergruppe aus Deutschland nach Südamerika gelangte. Die erste deutsche Kolonie wurde in Rio Grande do Sul im Jahre 1824 gegründet und zu Beginn des XX. Jahrhunderts wanderten die Personen erneut aus, diesmal von Brasilien nach Paraguay, und zwar in den Süden. 1900 wurde Hohenau gegründet, 1912 Obligado und 1918 Bella Vista. Diese drei Kolonien sind heutzutage als „Colonias Unidas “bekannt und liegen in Itapuá, Paraguay

Der vorliegende Aufsatz handelt von der Existenz verschiedener Eigenschaften des Hunsrückisch in dieser spezifischen Region Paraguays, in den ColoniasUnidas, im Vergleich zu denselben Eigenschaften, die im Hunsrückisch in der Region Rio Grande do Sul, in Brasilien erforscht wurden. Das Thema der Arbeit wurden anhand der früheren Erfahrungen der Autorin mit der Umgangssprache der ColoniasUnidas gewählt, in Anbetracht auf das Interesse, dass dasselbe für sie darstellt.

Die Methodologie, die angewandt wurde, um das Sprachmaterial zu erhalten, ist die Durchführung von Leitfadeninterviews mit Informanten aus den ColoniasUnidas. Das so entstandene Sprachmaterial wurde anschließend mit den sprachlichen Merkmalen des Hunsrückisch in Brasilien verglichen.

Das Hauptziel dieses Aufsatzes stellt die Erforschung von sprachlichen Merkmalen des hunsrückischen Dialekts in der Umgangssprache der Colonias Unidas dar. In Verbindung mit diesem Ziel wird auf die sprachlichen Eigenschaften des Hunsrückisch in Rio Grande do Sul eingegangen. Da die Behandlung der Thematik grundlegende Informationen über die Colonias Unidas voraussetzt, werden die geschichtlichen Hintergründe der Einwanderung und der historische Kontext der Gründung der Colonias Unidas kurz beschrieben.

\section{Allgemeine Beschreibung}

Anfang des 20. Jahrhunderts wurde Colonias Unidas im Süden Paraguays (Itapúa) durch deutschbrasilianische Einwanderer aus der Gegend von Rio Grande do Sul gegründet. Im Standesamt der Kolonie Hohenau kann man an den Dokumenten erkennen, dass die meisten Einwanderer aus der Gegend von Rio Grande do Sul kamen, da Geburtsorte wie 
Tacuara, Lageado, Estrela, Sao Sebastian do Caí, Caxias do Sul, Agudo, Montenegro und Teutonia in den Geburtsurkunden stehen (cf. Kegler 2000: 29). Die erste Kolonie, Hohenau, wurde im Jahr 1900 gegründet. Bald darauf entstanden die Nachbarkolonien Obligado (1912) und Bella Vista (1918).

Eine Tatsache, die für diese Arbeit wichtig erscheint, ist die ethnische Herkunft dieser Bewohner, die nicht ausschließlich auf Brasilien, sondern auf Europa zurückzuführen ist, woher die vieleImmigranten Brasiliens stammen. Anfang des 19. Jahrhunderts war Deutschland noch kein vereinigtes Land, sondern bestand aus Fürstentümern und Herzogtümern, in denen jedoch die deutsche Sprache mit ihrem jeweiligen Dialekt gesprochen wurde.

Die deutschen Einwanderer in der Gegend von Rio Grande do Sul stammten aus unterschiedlichen Regionen Deutschlands, unter anderem aus dem Hunsrück, einer Region in der Nähe des Rheins, wo der Hunsrückische Dialekt gesprochen wird. In Brasilien kamen die Einwanderer aus dem Hunsrück mit Deutschen aus den anderen Regionen, wie zum Beispiel Schwaben und Pommern, zusammen. So kam das Hunsrückisch in Kontakt mit mehreren anderen Dialekten. Außer ihrer jeweiligen Dialekte, sprachen die deutschen Einwanderer in Rio Grande do Sul auch Deutsch. In ihrem Aufnahmeland Brasilien bildeten die Einwanderer zu Beginn eine geschlossene Gruppe, die fast keinen Kontakt zu den Brasilianern pflegte, da sie sich in abgelegenen Gegenden niedergelassen hatte. Deshalb fehlte es auch an Interesse, die portugiesische Sprache in das Schulsystem einzuführen; sie funktionierte als linguafranca, die man erlernte, um mit den Einheimischen zu verhandeln. In den Schulen jedoch wurde vor allem auf Deutsch unterrichtet.

Die Migration nach Paraguay begann ungefähr 80 Jahre nachdem die ersten Einwanderer nach Brasilien gekommen waren. Sobald die Kolonie Hohenau gegründet worden war, entstanden die ersten deutschen Schulen, in denen die Kinder lesen, schreiben und rechnen lernten. Es muss hervorgehoben werden, dass eines der wichtigsten Angelegenheiten immer die Schulen waren. Sobald sich die Migranten etabliert hatten, wurde das Schulsystem aufgebaut. Als Lehrer wurden lokale Kräfte eingestellt; der Unterricht (von der ersten bis zur sechsten Klasse) fand in den ersten Jahren auf Deutsch statt, bis nach dem zweiten Weltkrieg die Schulen geschlossen wurden, und in das nationale Schulsystem eingliedert wurden (EscuelasNacionales). Bis dahin hatte der Spanischunterricht darin bestanden, Sätze von Deutsch auf Spanisch zu übersetzen. Nun wurde versucht, mehr Spanisch in den Unterricht einzuführen, wie aus den mit älteren Leuten geführten Gesprächen zu entnehmen ist. Trotzdem war es 1964 noch so, dass 40\% des Unterrichts auf Spanisch, und 60\% des Unterrichts auf Deutsch abgehalten wurde (cf. Warkentin 1998: 331).

Hinsichtlich der Sprache haben die deutschen Einwanderer nicht nur in Brasilien, sondern auch in Paraguay ihre Sprache und jeweiligen Dialekte beibehalten. Es ist jedoch zweifelhaft, ob die Einwohner der Colonias Unidas noch Hunsrückisch als eigenständigen Code sprechen. Auf die Befragung antworteten sie beispielsweise nicht „wir sprechen Hunsrückisch”, sondern sie sagten „Wir sprechen Deutsch “. Sie sind sich jedoch der Tatsache bewusst, dass sie kein Standarddeutsch oder, wie sie selbst angeben, ,richtiges "Deutsch sprechen. Wenn sie sich mit anderen Deutschen unterhalten, die erst seit kurzem im Land sind, oder zu Besuch verweilen, sagen sie, dass diese "feines Deutsch" oder "Hochdeutsch" sprechen, das sie selbst nicht beherrschen. Obwohl sie sich nicht als Hunsrückisch-Sprechende ausgeben, erkennen sie, wenn jemand Wörter oder Ausdrücke auf Hunsrückisch gebraucht, und kennen auch die Bedeutung jener Lexeme oder Sätze. Es ist also anzunehmen, dass in ihrer Sprache hunsrückische Formen vorhanden sind, deren sie sich beim Sprechen nicht immer bewusst sind. Diese Formen kann man bei Unterhaltungen mit 
verschiedenen Personen in unterschiedlichen Maßen wahrnehmen; daher unser Interesse nach hunsrückischen Merkmalen zu suchen.

Das Hauptziel diesesAufsatzesist folgendes:

- Sprachliche Merkmale des hunsrückischen Dialekts in der Umgangssprache der Colonias Unidasfestzulegen.

Dieses Hauptziel ist mit folgenden spezifischen Zielen verbunden:

- Beschreibung der sprachlichen Merkmale des Hunsrückisch in Deutschland. Brasilien.

- Beschreibung der sprachlichen Merkmale des Hunsrückisch in Rio Grande do Sul,

Mit sprachlichen Merkmalen sind nicht nur lexikalische, phonologische und morphologische Merkmale, sondern auch grammatische Eigenschaften gemeint. Da das Erkennen hunsrückischer Merkmale in der Sprache der Bewohner der ColoniasUnidas ${ }^{2}$ nur durch die Gegenüberstellung ihrer Sprache mit dem Hunsrückisch aus dem deutschen Sprachraum und dem in Brasilien gesprochenem Hunsrückisch möglich ist, werden wir uns sowohl mit dem herkömmlichen Hunsrückisch als auch mit dem Hunsrückisch in Rio Grande do Sul näher befassen.

Zur Erforschung der sprachlichen Merkmale sollen Gespräche mit Informanten aus den ColoniasUnidas geführt und anschließend analysiert werden. Dabei werden die lexikalischen, phonologischen, morphologischen und grammatischen Merkmale behandelt, die in dem Sprachmaterial auftreten und die man, im Vergleich zu den vorher beschriebenen Merkmalen des Hunsrückisch in Brasilien, letztendlich auch als hunsrückische Merkmale in den ColoniasUnidas einstufen kann.

\section{Methodologie}

Forschungsgegenstand der hier dargestelltenArbeit waren die sprachlichen Merkmale in der Umgangssprache der Bewohner der ColoniasUnidas, insbesondere solcher Merkmale die Informationen über den hunsrückischen Dialekt liefern. Es sollte jedoch zu Beginn ausdrücklich festgelegt werden, dass die Sprache der Einwohner von ColoniasUnidas bis heute unerforscht geblieben ist und dass in der vorliegenden Arbeit ein erster Versuch unternommen werden soll, die Merkmale des Hunsrückisch in den ColoniasUnidas zu identifizieren.

Die Sprachforschung des Hunsrückisch in Südamerika im Allgemeinen stellt ein noch ziemlich unergründetes Gebiet dar; es muss jedoch bemerkt werden, dass in Brasilien schon mehrere Studien zum Thema durchgeführt wurden (cf. Altenhofen 1995; Tornquist 1997) und dass die deutschstämmigen Brasilianer sich sehr um die Anerkennung und Erforschung ihres Dialektes bemühen. Da ColoniasUnidas im Süden Paraguays von deutschstämmigen Brasilianern aus Rio Grande do Sul gegründet wurde, stellen diese Studien eine entscheidende, grundlegende Quelle für unsere Arbeit dar. Umso mehr, da das Thema in Paraguay ein gänzlich unerforschtes Gebiet darstellt. Daher können wir uns auch nicht auf

\footnotetext{
${ }^{2}$ Online: http://www.duden.de/. (20.10.2011): Bezüglich der Volksbezeichnung (Ethnonym) der Bewohner der ColoniasUnidas, wird in dieser Arbeit versucht, den Termini „Kolonist “zu vermeiden, da dieses Wort auch die Bedeutung von „,kolonisieren “beinhaltet, also ,europäischer Siedler in einer Kolonie “, oder ,jemand, der kolonisiert “, und der Termini weder im Zusammenhang mit Europa noch mit Kolonisierung verstanden werden soll, sondern einfach nur als Selbstbezeichnung der Bevölkerung der ColoniasUnidas. Als Ersatz dafür wird von Siedlern oder von Immigranten, sowie von Bewohnern oder Einwohnern der ColoniasUnidas gesprochen.
} 
bereits formulierte Theorien oder angewandte Methoden berufen. Wenn ein Thema zum ersten Mal behandelt wird, verfügt der Wissenschaftler weder über Vorkenntnisse noch über widersprüchliche Theorien, die er eventuell gegenüberstellen könnte. Er hat sozusagen das Vergnügen, vieles falsch zu machen, ohne dass es jemand überprüfen kann. Es kann also nicht vom kumulativen Prinzip ausgegangen werden, das von Labov (1972) wie folgt formuliert wurde:

„The more is known about a language, the more we can find about it.“(Labov 1972: 98$)^{3}$

In dieser Arbeit wird versucht mit bestem Wissen und Gewissen Pionierarbeit zu leisten, um eine erste Annäherung zum Thema der sprachlichen Merkmale in der deutschen Umgangssprache der ColoniasUnidas zu geben.

Wenn es darum geht, Sprachmaterial zu sammeln, bedient sich die herkömmliche Dialektologie hauptsächlich der direkten oder der indirekten Methode. Die indirekte Methode besteht darin, schriftliche Befragungen durchzuführen; es werden Fragebögen an die Informanten verschickt und von ihnen selbst ausgefüllt. Ein eindeutiger Vorteil dieser Methode ist, dass das Ergebnis der Befragung nicht durch die Anwesenheit des Forschers beeinflusst wird; trotz dieses Vorteils wurde die Methode schon mehrfach kritisiert, da unter anderem keine Möglichkeit besteht, durch Rückfragen das Verständnis der Fragen abzusichern. Was aber die direkte Methode betrifft, wie es ihr Name schon vorahnen lässt, geht es um persönliches Sammeln der Informationen durch einen Befrager. Diese Art Methode bietet eindeutige Vorteile; sie gibt dem Interviewten die Möglichkeit, die Antworten ausführlicher zu gestalten (als das es bei schriftlichen Fragebögen möglich wäre) und der Forscher bekommt die Gelegenheit, auch aus nicht verbalen Kommunikationsmitteln und nicht verbalen Verhalten Schlüsse ziehen zu können (cf. Schlieben-Lange 1991: 122).

In dieser Arbeit wurde die direkte Methode angewandt und die Autorin selbst führte die Interviews. Es handelten sich hierbei um Leitfadeninterviews. Die Forscherin stellte während des Gesprächs mit dem Informanten ab und zu Fragen zu einem bestimmten Thema. Die Gespräche wurden auf ein Aufnahmegerät (Sony IC Recorder) aufgenommen, um nachher verschriftlicht zu werden. Das Ziel der Interviews war zweifach, nämlich die Alltagssprache der Informanten aufzuzeichnen und durch das Gespräch Informationen zum geschichtlichen Hintergrund der deutschbrasilianischen Migranten und der Gründung der ColoniasUnidas zu bekommen, die anschließend aufgearbeitet wurden.

Die Autorin hat diese Forschungsarbeit mit einer Explorationsphase begonnen, bevor die definitive Methodik festgelegt wurde. Die Explorationsphase bestand aus der TonAufzeichnung von kurzen Gesprächen mit verschiedenen Siedlern, die in den Monaten Juli bis August 2010 durchgeführt wurden. Die Gespräche warensogenannte Leitfadengesprächen. Als Leitfaden der Konversation wurden Fragen über die Herkunft, die Migration und die Gründung der Kolonie gestellt, wodurch die Interviewten kurz erklären konnten, wie und woher ihre Vorfahren nach Paraguay gelangt waren, und welche Erinnerungen sie noch von den ersten Zeiten der ColoniasUnidas durch die Erzählungen ihrer Eltern oder Großeltern bewahrten.

Während dieser Voruntersuchung konnte Sprachmaterial von folgenden Personen gesammelt werden:

\footnotetext{
3“Je mehr Kenntnisse man über eine Sprache hat, desto mehr kann man über sie finden”. (Übersetzung/DA).
} 


\begin{tabular}{|l|l|l|l|l|l|l|}
\hline Informanten & Geschlecht & Alter & Geburtsort & Wohnort & Beruf & Dauer \\
\hline Dietrich L. & Maskulin & 80 & Obligado & Obligado & Rentner & ca. 14' \\
\hline Karl R. & Maskulin & 72 & Hohenau & Hohenau & Rentner & ca. 24' \\
\hline Frida R. & Feminin & 55 & Bella Vista & Bella Vista & Lehrerin & ca. 19' \\
\hline Dorothea L. & Feminin & 73 & Obligado & Obligado & Rentnerin & ca. 9' \\
\hline Sara N. & Feminin & 50 & Obligado & Hohenau & Lehrerin & ca. 11' \\
\hline Udo F. & Maskulin & 82 & Obligado & Obligado & Rentner & ca. 15' \\
\hline Greta S. & Feminin & 86 & Bella Vista & Obligado & Rentnerin & ca. 11' \\
\hline Mirtha S. & Feminin & 93 & Brasilien & Obligado & Rentnerin & ca. 57' \\
\hline
\end{tabular}

Diese durchgeführten Interviews wurden als Explorationsphase eingestuft, da die Länge der Gespräche zu kurz war, um sprachliches Material zu analysieren. Dennoch konnte erkannt werden, dass die deutsche Sprache der Interviewten phonologische und lexikalische Merkmale des Hunsrückisch enthält. Man kann von einer Umgangssprache der ColoniasUnidas sprechen, die das Standarddeutsch mit verschiedenen Eigenschaften des Hunsrückischen verbindet. Infolgedessen wurde beschlossen, längere Interviews zu führen, mit Informanten die sich ungefähr im gleichen Alter befanden wie die Personen der Explorationsphase, und deren Vorfahren alle aus Brasilien, aus der Region Rio Grande do Sul stammten, auch wenn die Urahnen ursprünglich nicht immer aus der Region des Hunsrück in Deutschland kamen.

Die Gespräche für das eigentliche empirische Material sollten mindestens zwischen 45 und 60 Minuten andauern; die Informanten nur ältere Personen sein, da die Jüngeren das Deutsch nicht mehr so gut beherrschen und eher spanische Ausdrücke mit in die Konversationen bringen als Ältere. Alle Informanten sollten in den ColoniasUnidas geboren und ihre Vorfahren deutschbrasilianische Immigranten aus Rio Grande do Sul sein. Von der Autorin und Leiterin der Gespräche wurden einige Leitfragen formuliert, anhand derer die Informanten frei sprechen konnten. Das Hauptthema der Interviews war die Geschichte der ColoniasUnidas, da ein zweitrangiger Forschungsgegenstand dieser Arbeit der geschichtliche Hintergrund der Migranten war, die von Deutschland nach Brasilien und von Brasilien nach Paraguay gelangten.

Die Reihenfolge der Interviews wurde anhand des Alters der Informanten bestimmt, also angefangen bei dem/der Jüngsten/er, bis zum/zur Ältesten/er. Die Autorin entschied sich für dieses Kriterium, da alle anderen Merkmale sehr relativ waren, d.h. die Informanten lebten teilweise nicht mehr in der Stadt, in der sie geboren waren, oder waren sowieso in einer anderen Stadt aufgewachsen. Es kamen jedoch als Geburtsorte nur jene vor, die Teil der ColoniasUnidas sind: Hohenau, Obligado und Bella Vista. Die Auswahl der Informanten wurde anhand folgender Kriterien getroffen:

- Älter als 60 Jahre.

- In den ColoniasUnidas geboren sein.

- Vorfahren aus Brasilien, aus der Region Rio Grande do Sul.

- Deutsch sprechen.

FolgendeLeitfragen wurden während des Gesprächs befolgt:

1. Wo sind Sie geboren?

2. Welche Generation ist in Deutschland geboren?

3. Welche Generation ist in Brasilien geboren? Wo?

4. Wie war die Überfahrt von Deutschland nach Brasilien?

${ }^{4}$ Die Namen aller Informanten wurden zur Wahrung der Privatsphäre geändert. 
5. Wie war die Überfahrt von Brasilien nach Paraguay?

6. Was haben ihre Großeltern/Eltern mitgebracht?

7. Wie war die erste Zeit in Paraguay?

Was außer der Offenheit der Informanten noch hervorgehoben werden sollte, ist die Freundlichkeit und Hilfsbereitschaft, mit denen sie der Autorin entgegenkamen. Alle bemühten sich äußerst, soviel Details wie möglich zu nennen und viele führten ihre Fotoalben aus vergangenen Zeiten vor, oder zeigten Dokumente ihrer Großeltern bzw. Urgroßeltern, wie zum Beispiel Urkunden oder Schriftstücke.

Die Informanten deren Sprachproduktion schließlich in dieser Forschungsarbeit analysiert wurde, sind folgende:

\begin{tabular}{|l|l|l|l|l|l|l|l|}
\hline Informanten $^{\mathbf{5}}$ & Datum & Dauer & Geschlecht & Alter & Geburtsort & Wohnort & Beruf \\
\hline Walter R. & Juli 2010 & ca. 75, & Maskulin & 66 & Hohenau & Hohenau & Landwirt \\
\hline Britta P. & Juli 2011 & ca. 47 & Feminin & 69 & Bella Vista & Obligado & Rentnerin \\
\hline Siegfried R. & Juli 2011 & ca. 48 & Maskulin & 80 & Bella Vista & Obligado & Rentner \\
\hline Konrad G. & Juli 2011 & ca.44' & Maskulin & 81 & Hohenau & Hohenau & Rentner \\
\hline
\end{tabular}

Die Befragten gaben an, während ihrer Kindheit zu Hause nur Deutsch gesprochen zu haben und größtenteils erst in der Schule Spanisch gelernt zu haben. Aber durch den Umgang mit Nachbarn, die aus anderen Regionen Deutschlands stammen und somit auch andere Dialekte sprachen, hatten sie außerhalb der Schule und ihres Hauses auch Kontakt zu verschiedenen Dialekten, worunter das Schwäbische öfters genannt wurde. Alle Befragten haben die Schule besucht, obwohl sie sie mit unterschiedlichem Niveau abgeschlossen oder frühzeitig beendet haben. Alle wiesen mindestens Grundkenntnisse wie Lesen, Schreiben und Rechnen vor. Die meisten Informanten waren schon pensioniert, und einige kümmerten sich noch um ihre Ländereien.

DieDatenbearbeitung bestand folglich darin, die vier Audio-Dateien auf den Computer herunterzuladen, und orthographisch zu transkribieren. Anschließend sollten die Texte editiert werden, um nur das Sprachmaterial der Informanten vor Augen zu haben. Der nächste Schritt bestand darin, diese Texte systematisch nach spezifischen, hunsrückischen Merkmalen zu durchsuchen.

Die spezifischen Merkmale wurden in Tabellenform erstellt und anschließend systematisch analysiert und mit Merkmalen des Hunsrückisch in Brasilien verglichen. Die Ergebnisse werden im fünften Kapitel beschrieben, unter Beachtung der verschiedenen Beschreibungsebenen: die lexikalische, die phonologische und die grammatikalische Ebene.

\section{ERGEBNISSE}

\section{Phonologische Merkmale}

Im Korpus der vier Informanten konnten viele phonologische Merkmale festgestellt werden. In diesem Abschnitt soll bei der Analyse des Sprachmaterials der vier Informanten hauptsächlich auf solche Merkmale geachtet werden, die in der Fachliteratur als Hunsrückisch gelten und insbesondere in der Umgangssprache der Bewohner von Rio Grande do Sul in Brasilien auftreten. Verständnishalber werden die Beispiele in orthographischer Schreibweise gegeben, aber jeweils mindestens ein Beispiel phonologisch transkribiert.

\footnotetext{
${ }^{5}$ Die Namen aller Informanten wurden zur Wahrung der Privatsphäre geändert.
} 
An erster Stelle werden die vokalischen Merkmale behandelt und anschließend die konsonantischen, die im Hunsrückisch der ColoniasUnidas in Paraguay auftreten.

\section{Vokalische Merkmale}

In diesem Abschnitt werden alle phonologischen Eigenschaften des Hunsrückischen, die dem vokalischen Bereichentsprechen, aufgeführt. Es wurden fünf Merkmale erkannt, die im Folgenden beschrieben werden. All diese Beispiele traten im Korpus der vier Informanten auf.

\section{Entrundung: $[y]>[i]$}

Im Hunsrückisch in Rio Grande do Sul kann man die Eigenschaft der Entrundung oder Delabialisierung von Vokalen feststellen. In unserem Sprechmaterial, das auf Gespräche mit Informanten von Colonias Unidas basiert, kann man das Auftreten von entrundeten Vokalen ebenfalls beobachten. Der gespannte Vokal ü wird im Hunsrückisch entrundet (delabialisiert) und als $\mathbf{i}$ ausgesprochen, das heißt nicht als [y], sondern als [i:] artikuliert. Wir übernehmen die leserfreundliche Gestaltung der Umschreibung von Tornquist (1997), indem wir einen doppelten Vokal benutzen (in diesem Fall ii), um lange Vokale orthographisch zu kennzeichnen, wie in folgenden Beispielen: riber[ri:bá] $\sqcap-$ rüber [rybá], Stick - Stück, Brida - Brüder.

\section{Entrundung: [ø] $>[e:]$}

Ebenfalls der im deutschen gerundete Vokal ö kommt bei drei der vier Informanten als entrundeter Vokal vor: anstatt das Wort schön [Øø:n]wie in dem Standarddeutsch auszusprechen, sagen sie scheen[fe:n]. Dies ist ein weiteres Merkmal der hunsrückischen Sprechweise in Rio Grande do Sul, die in den Colonias Unidas auftritt. Wie in der o.g. Form der Entrundung [y] > [i:], verwenden wir auch hier die Umschreibung Tornquists (1997), indem wir für lange Vokale die Doppelschreibung anwenden (in diesem Fall $\boldsymbol{e e}$ ). Die Entrundung (Delabialisierung) wurde in den folgenden Formen beobachtet: eefentlichen [EfentlI $\square]$ - öfentlichen[œfentlI $\sqcap]$, scheen - schön, kennt - könnt.Im Allgemeinen tritt das Merkmal der Entrundung [ø] und [œ] > [e:] nicht so oft auf wie die Entrundung von $[\mathrm{y}]>[\mathrm{i}:]$.

\section{Vokalhebung von [o] > [u]}

Die Vokalhebung bzw. Vokalsenkung sind Eigenschaften, die im Hunsrückisch in Rio Grande do Sul auftreten, und die wir auch in den Gesprächen mit Informanten aus den Colonias Unidas verzeichnen konnten. Man kann, in vermindertem Maße, in dem Sprachmaterial das Merkmal der Vokalhebung bemerken, zum Beispiel bei der Vokalhebung von o zu u, z.B. sugar [zuүa].

\section{Vokalsenkung von $[i]>[e]$ und von $[u]>[o]$}

Ein weiteres Merkmal betrifft die mittel und niederdeutsche Senkung, bei der der hohe Vokal i gesenkt wird und zu einem e wird. Ein Beispiel dafür ist die standarddeutsche Form Kirche [kIrçe], die im Sprachmaterial als Kerch [kerç] auftritt; dieses Beispiel tritt bei dem Informant Siegfried R. einmal auf. Die Senkung kommt auch bei hinteren Vokalen vor: das hohe gespannte [u] oder das ungespannte [v] wird zu [o] oder zu [?]. Beispielweise wird die standarddeutsche Form durch [dvr】] von dem Informant Siegfried R. zweimal als[d]̣rç] (dorch) ausgesprochen. Dieses Merkmal ist kein kategorisches Merkmal, da der Wechsel der Vokale nicht immer stattfindet und nicht bei allen vier Informanten bemerkt werden kann. 


\section{Das auslautende -e}

Das Fehlen des auslautenden Vokals $-\mathbf{e}$ ist eine Eigenschaft, die im Hunsrückisch in Brasilien verzeichnet wird und die im Sprachmaterial der Colonias Unidas ebenfalls bemerkt wurde.

In den Gesprächen mit Informanten entfällt öfters das unbetonte -e im Auslaut, sowohl bei Nomen im Singular und Plural als auch bei Verben in der 1. Person Singular (im Präsens) und bei der 1.und 3. Person im Singular (im Präteritum), zum Beispiel: Junge [jo $\sqcap$ e] das wie Jung [jo k], hätt - hätte, Schul - Schule

Obwohl dieses Merkmal häufig auftritt, kann nicht behauptet werden, dass es kategorisch ist. In vielen Fällen sprechen die Informanten das auslautende $-\mathbf{e}$ doch aus.

\section{Monophthongierung}

Eine andere Eigenschaft des Hunsrückisch in Brasilien, die im Sprachmaterial der Colonias Unidas wiedergefunden werden konnte, ist die Monophthongierung, die stattfindet, wenn ein Doppelvokal zu einem einfachen Vokal wird, wie zum Beispiel der mhd. Diphthong ou, der bei den Riograndenser-Sprechern manchmal alsoo realisiert wird. Bei dem Gespräch mit dem Informant Siegfried R. konnte eine ähnliche Monopthongierung gefunden werden, nämlich beim Nomen Aufgabe [awfgabə], dasder Informant als Uffgabe [vfgabə] aussprach; hier fand die Monophthongierung von [aw] zu [v]statt. Das Merkmal kann nicht als kategorisch bezeichnet werden, da wir nur ein Beispiel im Sprachmaterial von Siegfried R. finden konnten.

\section{Schlussfolgerung zu Vokalen}

Im Vergleich zum Hunsrückisch, das in Brasilien gesprochen wird und dessen Merkmale als Vorlage für diese Analyse gelten, sind bestimmte Unterschiede festzustellen. Das Merkmal, das bei den Gesprächen mit Informanten aus den Colonias Unidas in dem vokalischen Bereich am meisten vorkommt, ist die Entrundung der Vokale von ü auf ii oder i, und von $\ddot{0}$ aufee. Merkmale die in Rio Grande do Sul auftreten, wie Vokalsenkung bzw. Vokalhebung (Vokale von i zu e, und von u zu o,oder von o zu u), die Diphthongierung bzw. Monophthongierung, von [aw] zu [v] ), oder das Verschwinden des -e am Ende von Nomen (oder Verben), sind in den durchgeführten Gesprächen in verringertem Maße auffindbar. Im Vergleich zum Riograndenser-Hunsrückisch in Brasilien, in dem diese sprachlichen Eigenschaften angeblich fast kategorisch sind, treten in der Sprechart der Colonias Unidas keine dieser vokalischen Eigenschaften als kategorische Merkmale in unserem Sprachmaterial auf. Außerdem muss erwähnt werden, dass nicht alle Merkmale zu beobachten sind. So kommt beispielsweise die Vokaldehnung oder Vokalkürzung überhaupt nicht vor.

\section{Konsonantische Merkmale}

In diesem Abschnitt werden die phonologischen Merkmale des Hunsrückisch im konsonantischen Bereich erfasst und mit dem brasilianischen Hunsrückisch verglichen. Insgesamt kann man drei Eigenschaften für Konsonanten bemerken.

\section{Das intervokalische und auslautende $g$}

Das zwischen Vokalen oder am Wortende auftretendeg ist nicht nur eine Eigenschaft des brasilianischen Hunsrückisch, es wurde auch in unserem Korpus aufgefunden, jedoch nur am Wortende. In diesem Kontext wird das g nicht verhärtet ausgesprochen, d.h. als [k], sondern als ich-bzw. ach- Laut, z.B. Teig [taIç], daher auch Teich geschrieben. Folgende Wörter sind Beispiele: Kriech [kriף- Krieg [krik], Teich - Teig. 
In diesen Beispielen tritt das auslautende $\mathbf{g}$, das in der Standardvarietät als [k] ausgesprochen wird, als ich- Laut auf, und wird am Wortende als [ç] ausgesprochen, d.h. als palataler ${ }^{6}$ Frikativlaut. Diese Aussprache konnte man oft im Sprachmaterial bemerken.

Folgende Beispiele beziehen sich auf dasselbe Merkmal wie oben, mit dem Unterschied, dass das $\mathbf{g}$ am Wortende nicht wie ein ich-Laut, sondern wie ein ach-Laut ausgesprochen wird. Das phonetische Symbol [x] steht für den velaren ${ }^{7}$ Frikativlaut: Geburtstach - Geburtstag.

\section{Die zweite Lautverschiebung}

Ein weiteres Merkmal des Hunsrückisch in Brasilien, das in den Colonias Unidas auftritt, ist die zweite Lautverschiebung, die in der Veränderung der Verschlusslaute $\mathbf{p}, \mathbf{t}, \mathbf{k}$ zu pf/f(f), ts/s(s) bzw. k/chbesteht. Diese Verschiebungen wurden je nach Position im Wort nur teilweise durchgeführt. Einige Beispiele, die die nicht durchgeführte zweite Lautverschiebung bezeugen, wurden auch während der Interviews in den Colonias Unidas vermerkt: Kop [kop]- Kopf [kJpf], stampen - stampfen, Firsiche - Pfirsiche.

\section{Das auslautende -n}

Eine Eigenschaft des Hunsrückisch in Brasilien stellt das -n dar, dass am Ende eines Nomens oder am Ende von Verben im Infinitiv, in der 1. oder 3. Person Plural (im Präsens), sowie im Partizip Perfekt wegfällt. Dieses Merkmal konnte bei dem Informant Siegfried R. in dem Wort Autoche bemerkt werden. Folglich artikulieren alle vier Informanten sozusagen immer das auslautende $-\mathbf{n}$.

\section{Schlussfolgerung zu Konsonanten}

Im Allgemeinen kann bemerkt werden, dass der Sprachproduktion in Colonias Unidas weniger konsonantische als vokalische Merkmale vorkommen. Die konsonantischen Merkmale, die identifiziert wurden, treten nie systematisch oder kategorisch auf. Auch kommen nicht bei allen Informanten vor: so produziert zum Beispiel Konrad G. keines der Merkmale. Bei ein und demselben Informanten kommen sie aleatorisch vor, zum Beispiel braucht Siegfried R. für die Bedeutung „Weg“manchmal die standartdeutsche Form Weg [wek], manchmal die hunsrückische Form Wech [weç]. In den Interviews treten folglich hunsrïckische Merkmale immer zusammen mit standarddeutschen Merkmalen auf.

Ein typisches Merkmal, das sehr oft im Korpus vorkommt, ist gerade jenes, bei dem das orthographisch repräsentierte $\mathbf{g}$ als [ç] ausgesprochen wird, sowohl in der Mitte als auch am Ende des Wortes. Ein anderes Merkmal, das ebenfalls oft auftritt, stellt die zweite Lautverschiebung dar, die im Hunsrückisch nicht durchgeführt wurde (Pfirsiche>Firsiche).

Im Vergleich zum Riograndenser Hunsrückisch gibt es Merkmale, die in unserem Korpus nicht erscheinen, wobei nicht behauptet werden kann, dass sie in der Sprache der Colonias Unidas nicht bemerkt wurden. So kommt der Konsonantenzusammenfall nicht vor, in dem das $\mathbf{p}$ stimmhaft ausgesprochen wird (Paar>Baar). Der Verschlusslaut b, der als [v] ausgesprochen wird (lieber>liewer), und die Assimiliation von nd und nt $\mathrm{zu}$ nn (Kinder Kinner) wurden ebenfalls nicht beobachtet. Ein anderes Merkmal, das nicht vorkommt, ist der sch-Laut in Konsonantenverbindungen, zum Beispiel bist>bischt. Auf konsonantischer Ebene gibt es also mehrere hunsrückische Merkmale, die in den Colonias Unidas nicht beobachtet wurden.

Die Merkmale, die in den Gesprächen verzeichnet werden konnten, sind mehr oder weniger unbewusst von den Informanten erzeugt worden. Sie verwendeten das hunsrückische

\footnotetext{
${ }^{6}$ Palatale Laute werden im harten Gaumen produziert.

${ }^{7}$ Velare Laute werden im weichen Gaumen produziert.
} 
Merkmal spontan bzw. unbewusst, da sie sich selbst korrigierten (Hyperkorrektur), wenn ihre Aussprache ihrer Meinung nach, falsch 'klang.

Es kann also behauptet werden, dass in den ColoniasUnidas phonologische Eigenschaften des Hunsrückisch in der Sprechweise der älteren Leute zwar anwesend sind, aber das Hunsrückisch nicht als eigenständiges Sprachsystem ausgeprägt ist. Die Merkmale treten in der Umgangssprache auf, d.h. in einer Sprechweise, die zum Standarddeutschen tendiert, im Gegensatz zu Rio Grande do Sul, wo das Hunsrückisch angeblich als separate Varietät gesprochen wird.

\section{Morphologische und syntaktische Merkmale}

Was die Eigenschaften des Hunsrückisch auf morphologischer und syntaktischer Ebene betrifft, konnten nicht viele Beispiele im Sprachmaterial der Informanten der Colonias Unidas aufgedeckt werden. Insgesamt sind es zwei Merkmale, die hier aufgeführt werden sollen.

\section{Akkusativ-Ergänzungen}

Das grammatikalische Hauptmerkmal, dass das Hunsrückisch charakterisiert, ist die Benutzung des Akkusativs statt des Dativs. Die Ergänzungen der Verben werden beinahe ausnahmslos in den Akkusativ gesetzt, auch nach Präpositionen, die im Standarddeutschen das Dativ-Objekt anzeigen. Zum Beispiel: mit dich - mit dir, ich gib dich - ich gebe dir. Dieses Merkmal kommt nicht bei allen Informanten vor. Zu beachten ist, dass bei dieser Eigenschaft die Informanten weniger Hyperkorrekturen vornehmen als bei den phonologischen Merkmalen.

\section{Kein Wechsel von $[a]>[\ddot{a}]$ und $[e]>[i]$}

Ein Aspekt der im Hunsrückisch in Brasilien sowohl in der 2. und 3. Person im Singular Präsens als auch im Imperativ Singular auftritt, aber in dem Sprachmaterial der Colonias Unidas nur in der 3. Person im Singular Präsens bemerkt werden konnte, ist die nicht vollzogene Änderung von [a] > [ä] und [e] >[i]. Zum Beispiel bei Britta P. konnte beobachtet werden, dass sie die Verbform liest wie lest ausspricht; der Informant Walter R. spricht die Form sieht wie seht aus. Die Vokalveränderung, die typisch für das Standdardeutsch ist (ich sehe; du siehst; er/es/sie sieht) findet im Hunsrückisch nicht statt (ich sehe; du sehst; er/es/sie seht). Dieses Merkmal kommt jedoch nicht bei allen Informanten vor und wird selbst bei einem Informanten nicht in allen in Frage kommenden Spracheinheiten realisiert.

\section{Schlussfolgerung zu morphologischen und syntaktischen Merkmalen}

Die ausgeprägteste Eigenschaft, die sich in den Gesprächen äußert und die mit den Merkmalen des Hunsrückisch in Rio Grande do Sul übereinstimmt, ist die Benutzung des Akkusativ-Objekts in fast allen Fällen, in denen die deutsche Standardvarietät ein DativObjekt verlangt.

\section{Lexikalische Merkmale}

Das Auftreten von bestimmten Lexemen hängt eng mit den Themen zusammen, die in den Interviews angesprochen wurden. Da es sich um Leitfadeninterviews mit offenen Fragen handelt, die die Autorin selbst stellt, ist die Antwort der Informanten in bestimmtem Maße mit den gestellten Fragen verbunden. Die Wahl des Themas „Geschichte“ geschah in der Annahme, dass die Informanten viel über den damaligen Alltag erzählen würden und in diesem Kontext hunsrückische Ausdrücke benutzen würden. Dadurch, dass die Informanten sich mehr oder weniger bemühten, ,gutes' Deutsch zu sprechen, verringerte sich die 
Möglichkeit in den circa 45 minutenlangen Interviews viele hunsrückische Lexeme zu realisieren. Wahrscheinlich hätte die Menge des Sprachmaterials dazu etwas umfassender sein sollen. Trotz dieser Tatsachen konnte in dem Sprachmaterial eine aussagekräftige Menge an lexikalischen Merkmalen erkannt werden, wie an den folgenden Beispielen zu erkennen ist:

\begin{tabular}{|c|c|c|c|}
\hline Lexem & Äußerung & Standartdeutsch & Informant \\
\hline ------- & ----------------- & ------- & Britta P. \\
\hline $\begin{array}{l}\text { - Wäsche } \\
\text { - maie gang } \\
\text { - sin jo gang } \\
\text { - rangier mich } \\
\text { - hoch }\end{array}$ & $\begin{array}{l}\text { - Er hat sich ordentliche Wäsche gekauft } \\
\text { - Ich geh jetzt maie } \\
\text { - Ich sin jo gang } \\
\text { - Ich rangier mich mal } \\
\text { - ...so ein hoher, blonder Bursche }\end{array}$ & $\begin{array}{l}\text { - Kleidung } \\
\text { - spazieren gehen } \\
\text { - ich bin gegangen } \\
\text { - ich mache mich } \\
\text { fertig } \\
\text { - groß }\end{array}$ & Konrad G. \\
\hline $\begin{array}{l}\text { - Uffgabe } \\
\text { - Guri } \\
\text { - abgeschwat } \\
\text { - Autoche } \\
\text { - Ranchoche } \\
\text { - hupsen } \\
\text { - mir }\end{array}$ & $\begin{array}{l}\text { - Man musst die Uffgabe mache } \\
\text { - ...wie so kleine Guri, musst man in die } \\
\text { Plantasch... } \\
\text { - Wenn man frech war, habn se uns } \\
\text { abgeschwat } \\
\text { - Das kleine Autoche, alle drauf } \\
\text { - ...und sonRanchoche gebaut, } \\
\text { sonHäussche... } \\
\text { - Zwei Meter leicht weiter gehupst } \\
\text { - Mir konnten auch kein Spanisch }\end{array}$ & $\begin{array}{l}\text { - Aufgabe } \\
\text { - Kinder } \\
\text { - Verhauen } \\
\text { - ein kleines Auto } \\
\text { - ein kleines } \\
\text { Farmhaus } \\
\text { - hüpfen } \\
\text { - wir }\end{array}$ & $\begin{array}{l}\text { Siegfried } \\
\text { R. }\end{array}$ \\
\hline $\begin{array}{l}\text { - hupsen } \\
\text { - ehnta } \\
\text { - mir }\end{array}$ & $\begin{array}{l}\text { - Und der hupstriiba... } \\
\text { - ...isfriih gestorben, ehnta als ihre Mutter } \\
\text { - Mir haben vierhundert Jahre Geschichte } \\
\text { zurück }\end{array}$ & $\begin{array}{l}\text { - hüpfen } \\
\text { - früher } \\
\text { - wir }\end{array}$ & Walter R. \\
\hline
\end{tabular}

Sowohl bei dem Informanten Konrad G. als auch bei Siegfried R. kommen mehrere lexikalische Formen vor, die der hunsrückischen Sprechweise zugeschrieben werden. Bei Britta P. konnte während des Gesprächs kein Merkmal lexikalischer Art festgestellt werden.

Ein Wort, das nicht nur bei Siegfried R., sondern auch bei Walter R. auftritt, ist das Verb hupsen („hüpfen“), und das Pronomen wir, das beide als mir aussprechen. Bei den anderen zwei Informanten treten diese Formen nicht auf. Beide Merkmale kommen auch im Hunsrückisch in Brasilien vor.

Es ist zu beachten, dass diese Formen nicht sofort am Anfang des Gesprächs auftraten, sondern eher nachdem die Informanten ihre anfängliche Scheu überwunden hatten und vom formalen Register in spontanere Redensart übergingen.

Diese Ergebnisse zeigen, dass hunsrückische Lexeme noch im Usus sind, da trotz der kurzen Gesprächsdauer bei drei Informanten relativ viele Beispiele vorkamen. Es ist anzunehmen, dass die Informanten selbst diese Formen als deutsche Wörter ansehen, obwohl zum Beispiel zwei von ihnen, Konrad G. und Siegfried R., ausdrücklich verschiedene Lexeme als Hunsrückisch bekundeten. Auch ist zu erwähnen, dass während der Gespräche niemand seine Ausdrucksweise hinsichtlich der Wahl der Lexeme korrigierte; die drei Informanten, die hunsrückische Lexeme benutzten, gebrauchten sie spontan.

$\mathrm{Da}$ die Autorin in den Colonias Unidas aufgewachsen ist, konnte sie schon vor Langem sprachliche Merkmale der dortigen Umgangssprache bemerken, die den hunsrückischen Formen entsprechen, vor allem auf lexikalischer Ebene. Diese Merkmale tauchen $\mathrm{ab}$ und zu unbewusst in Alltagsgesprächen auf; die Autorin hatte schon öfters die Leute gefragt, ob sie wissen, woher diese Merkmale kommen, ohne eindeutige Antworten zu erhalten. 
Mit dem Ziel, mehr Informationen zu diesem Aspekt zu bekommen, wurde der Entschluss gefasst, dieselben Informanten noch einmal anhand einer Liste hunsrückischer Wörter zu befragen. Eine Liste von 24 Wörtern wurde anhand der in Tornquist (1997) enthaltenen Informationen zum Hunsrückisch in Brasilien aufgestellt. In den deutschbrasilianischen Gemeinden sollen folgende hunsrückischen Wörter noch benutzt werden:

\begin{tabular}{|l|l|}
\hline Hunsrückisch in Rio Grande do Sul & Standarddeutsch \\
\hline als (er kommt als bei uns) & ab undzu \\
\hline Uffgabe & Aufgaben \\
\hline Panz & Bauch \\
\hline Motzig & Beleidigt \\
\hline Stolz & die "schicke" Frau \\
\hline Leppsch & fad, geschmacklos \\
\hline ehnder & Früher \\
\hline Hinkel & Huhn \\
\hline hupsen & Hüpfen \\
\hline Hämmes & Kalb \\
\hline Guri & Kinder \\
\hline Wäsche & Kleidung \\
\hline Schmier & Marmelade \\
\hline Maul & Mund \\
\hline Gaul & Pferd \\
\hline Buckel & Rücken \\
\hline schappich & Schlechtdransein \\
\hline Käs-Schmier & Schmier-Käse \\
\hline Sau (oderMoog) & Schwein \\
\hline schickdich & Sichbenehmen \\
\hline maiegehn & Sichgegenseitigbesuchen \\
\hline Dibbe/ Tippe & Topf \\
\hline grinsig & Unzufrieden \\
\hline abschwaten & Verhauen \\
\hline
\end{tabular}

Die Informanten wurden einzeln befragt, ob sie diese Wörter kennen, ohne deren Bedeutung auf Deutsch bekanntzugeben. Jedes Lexem bzw. jeder Ausdruck wurdevorgelesen, und der Informant bejahte oder verneinte, das Wort zu kennen. Was ihre Bedeutung anbetrifft, sind es Wörter, die hauptsächlich im Alltag benutzt werden, also Tiernamen, Gemütszustände, Körperteile und einige Aktivitäten, sowie Gegenstände aus der Küche. Das Ergebnis dieser Befragung sieht wie folgt aus.

\begin{tabular}{|l|l|l|l|l|l|}
\hline $\begin{array}{l}\text { Hunsrückich Rio } \\
\text { Grande do Sul }\end{array}$ & Standartdeutsch & $\begin{array}{l}\text { Britta } \\
\text { P. }\end{array}$ & $\begin{array}{l}\text { Konrad } \\
\text { G. }\end{array}$ & $\begin{array}{l}\text { Siegfried } \\
\text { R. }\end{array}$ & $\begin{array}{l}\text { Walter } \\
\text { R. }\end{array}$ \\
\hline als (er kommt als bei uns) & ab undzu & X & X & X & X \\
\hline Uffgabe & Aufgaben & X & X & X & X \\
\hline Panz & Bauch & X & X & X & X \\
\hline Motzig & Beleidigt & X & X & X & X \\
\hline stolz & die "schicke" Frau & X & X & X & X \\
\hline leppsch & fad, geschmacklos & X & X & X & X \\
\hline ehnder & früher & X & X & X & X \\
\hline Hinkel & Huhn & X & X & X & X \\
\hline hupsen & hüpfen & X & X & X & X \\
\hline Hämmes & Kalb & X & X & X & X \\
\hline
\end{tabular}




\begin{tabular}{|c|c|c|c|c|c|}
\hline Guri & Kinder & X & $\mathrm{X}$ & $\mathrm{X}$ & $\mathrm{X}$ \\
\hline Wäsche & Kleidung & $\mathrm{X}$ & $\mathrm{X}$ & $\mathrm{X}$ & $\mathrm{X}$ \\
\hline Schmier & Marmelade & $\mathrm{X}$ & $\mathrm{X}$ & $\mathrm{X}$ & $\mathrm{X}$ \\
\hline Maul & Mund & $\mathrm{X}$ & $\mathrm{X}$ & $\mathrm{X}$ & $\mathrm{X}$ \\
\hline Gaul & Pferd & $\mathrm{X}$ & $\mathrm{X}^{*}$ & $\mathrm{X}$ & $\mathrm{X}$ \\
\hline Buckel & Rücken & $\mathrm{X}$ & $\mathrm{X}$ & $\mathrm{X}$ & $\mathrm{X}$ \\
\hline schappich & schlechtdransein & $\mathrm{X}$ & $\mathrm{X}$ & $\mathrm{X}$ & $\mathrm{X}$ \\
\hline Käs-Schmier & Schmier-Käse & $\mathrm{X}$ & $\mathrm{X}$ & $\mathrm{X}$ & $\mathrm{X}$ \\
\hline Sau (oderMoog) & Schwein & $\mathrm{X}$ & $\mathrm{X}$ & $\mathrm{X}$ & $\mathrm{X}$ \\
\hline schickdich & sichbenehmen & $\mathrm{X}$ & $\mathrm{X}^{* *}$ & $\mathrm{X}$ & $\mathrm{X}$ \\
\hline maiegehn & $\begin{array}{l}\text { sichgegenseitigbesuche } \\
\mathrm{n}\end{array}$ & $\mathrm{X}$ & $\mathrm{X}$ & $\mathrm{X}$ & $\mathrm{X}$ \\
\hline Dibbe/ Tippe & Topf & Tippe & Tippe & Tippe & Tippe \\
\hline grinsig & unzufrieden & $\mathrm{X}$ & $\mathrm{X}$ & $\mathrm{X}$ & $\mathrm{X}$ \\
\hline abschwaten & verhauen & $\mathrm{x}$ & $\mathrm{X}$ & $X$ & $X$ \\
\hline
\end{tabular}

* Informant G. gab an, dass im Hunsrückisch das Wort Klepper für „Pferd“ benutzt wird.

**Informant gab die Pluralform schickt euch an.

Wie aus dieser Tabelle hervorgeht, erkannten alle vier Informanten die gegebenen Formen als hunsrückische Formen. Sie kannten auch deren Bedeutung, wie beispielsweise Konrad G., der klar zu verstehen gab, dass das Wort Klepper („Pferd“) ebenfalls Hunsrückisch sei, nachdem er $\boldsymbol{G a u l}$ auch als hunrückisches Lexem deutete. Die Form Klepper wird weder in Tornquist (1997) noch in Altenhofen (1995) erwähnt. Derselbe Informant lieferte uns weitere Sprachdaten zum Hunsrückisch, in diesem Fall grammatikalischer Art, als er erklärte, dass der hunsrückische Ausdruck schick dich auch im Plural schickt euch benutzt wird. Mit diesem Beitrag wollte er die ihm vorgestellte Liste erweitern. Die anderen Informanten beschränkten sich darauf, zu bestätigen, dass sie die Wörter kennen.

Die Informantin Britta P. sprach jedoch während der Befragung die Rolle der Kontaktsituation des Hunsrückischen mit anderen Sprachen an, da ihre Vorfahren nicht aus dem Hunsrück stammen, aber sie selbst all diese Lexeme erkannte und kannte, da in Rio Grande do Sul und in ColoniasUnidas der Kontakt zu dem Hunsrückisch stattgefunden hatte.

Anhand dieser letzten Befragung konnte bewiesen werden, dass der hunsrückische Wortschatz als solcher bekannt ist, auch wenn nicht bewiesen wird, inwieweit er im Alltag angewandt wird. Auf Grund der spontanen und schnellen Bejahung der Fragen ist jedoch anzunehmen, dass die älteren Einwohner der ColoniasUnidas die Wortformen benutzen, auch wenn sie nicht im Korpus auftreten. Von Bedeutung ist, dass sogar Britta P. und Walter R., deren Vorfahren nicht aus dem Hunsrück in Deutschland stammten, wohl aber aus Rio Grande do Sul, die lexikalischen Merkmale in (9) als Hunsrückisch ansahen.

\section{SCHLUSSFOLGERUNG}

Das Hunsrückisch ist ein wichtiger Bestandteil des kulturellen Lebens in Rio Grande do Sul. Durch die Übersiedlung der Deutschbrasilianer vor mehr als hundert Jahren in den Süden Paraguays, wo sie die Colonias Unidas gründeten, ist das Hunsrückisch auch hier erhalten geblieben und ist eine Komponentedieser Gemeinschaft geworden.

Das Auftreten von hunsrückischen Merkmalen in den Colonias Unidas konnte anhand von Gesprächen mit Informanten aus Obligado, Hohenau und Bella Vista bestätigt werden. Die Gespräche, die das Sprachmaterial der vorliegenden Forschungsarbeit darstellen, wurden teils auf Standarddeutsch teils in der Umgangssprache geführt. Im Sprachmaterial konnten 
phonologische, morphologische und syntaktische Merkmale des Hunsrückisch festgestellt werden, sowie lexikalische Merkmale, die dem Hunsrückisch entsprechen.

Das Sprachmaterial wurde in Hinsicht auf Merkmale des Hunsrückisch in Brasilien verglichen, da unsere Information bezüglich der Merkmale des Hunsrückisch in Deutschland sehr reduziert war.

Es wurden hauptsächlich phonologische Merkmale des Hunsrückisch in der Umgangssprache der Colonias Unidas aufgedeckt, sowohl im vokalischen als auch im konsonantischen Bereich. Dabei konnten Beispiele bei den Informanten der Colonias Unidas festgestellt werden, in denen die Merkmale des Hunsrückisch in Brasilien zu erkennen sind. Aber auch morphologische, syntaktische und lexikalische Merkmale traten auf, jedoch in verminderter Menge. Die Beispiele, die für die verschiedenen Merkmale auftraten, waren nicht kategorisch. Die Informanten verbesserten öfters ihre Aussprache. Trotzdem kann gesagt werden, dass es eindeutig hunsrückische Merkmale waren.

Die Umgangssprache der ColoniasUnidas stellt keinen selbständigen Code dar, sondern ist von dialektalen Merkmalen des Hunsrückisch geprägt. Dies will heißen, dass sprachliche Interaktionen nicht ausschließlich auf Hunsrückisch stattfinden, sondern dass im Standarddeutschen und/oder in der Umgangssprache hunsrückische Merkmale auftreten. Durch die Gegenüberstellung mit dem Hunsrückisch in Brasilien, anhand der in den Forschungsarbeiten von Altenhofen und Tornquist gelieferten Daten, konnte dies für die unterschiedlichen Sprachebenen bewiesen werden.

Die grundlegende Schlussfolgerung dieser Arbeit ist daher die Tatsache, dass die Bewohner der Colonias Unidas noch heutzutage, über hundert Jahre nach der Gründung von Hohenau, Obligado und Bella Vista, weiterhin Merkmale des Hunsrückisch in ihrer Umgangssprache aufweisen.

\section{Bibliographie}

Altenhofen, Cléo V. (1995): Hunsrückisch in Riogrande do Sul.Ein Beitrag zur Beschreibung einer deutschbrasilianischen Dialektvarietät im Kontakt mit dem Portugiesischen. InauguralDissertation zur Erlangung des Akademischen Grades eines Dr. phil., vorgelegt der Johannes Gutenberg-Universität zu Mainz.

Bausinger, Hermann (1972): Deutsch für Deutsche. Wiesbaden, Munzelbücher.

Bergmann, Günther J. (1994): Auslandsdeutsche in Paraguay, Brasilien, Argentinien. Bad Münstereisfel, Westkreuz.

Dittmar, Norbert (1973): Soziolinguistik. Frankfurt am Main, Athenaeum.

Kegler, Roland (2000): 1900- 2000 Hohenau. Cien años en las altas praderas. Hohenau, CigramImprenta-Editorial.

Labov, William (1972): "SomePrinciples of Linguistic Methodology". Language in Society 1,1: $97-$ 120.

Polenz, Peter von (1972): Geschichte der deutschen Sprache. Berlin, Walter de Gruyter.

Schlieben-Lange, Brigitte ([1973] 1991 $\left.{ }^{3}\right)$ : Soziolinguistik. Eine Einführung. Stuttgart/Berlin/Köln, Kohlhammer.

Tornquist, Ingrid Margareta (1997): „Das hon ich von meiner Mutter“. Zu Sprache und ethischen Konzepten unter Deutschstämmigen in Rio Grande do Sul. Acta Universitatis Umensis, Umeå Studies in the Humanities 137, Monograph.

Vater, Heinz (2002): Einführung in die Sprachwissenschaft. München, Wilhelm Fink Verlag.

\section{Sekundärliteratur}

Altenhofen, Cléo V.; Frey, Jacqueline; Käfer, Maria L.; Klassmann, Mário; Neumann, Gerson R.; Pupp Spinassé, Karen (2007): "Fundamentosparaumaescrita do Hunsrückisch faladono Brasil". RevistaContingencia, Volumen 2. 
Aubin, Hermann.; Frings, Theodor.; Müller, Josef (1926): Kulturströmungen und Kulturprovinzen in den Rheinlanden, Bonn, Ludwig Rohrscheid.

Diener, G. Walter (1988): HunsrückerWörterbuch. Liechtenstein, Vaduz.

Fausel, Erich (1959): Die deutschbrasilianischeSprachmischung. Probleme. Vorgang und Wortbestand. Berlin, Schmidt.

Finke, Theodor (1989): Leben und Arbeit deutschsprachigerSiedler in Südbrasilien und Paraguay. Bremen, Jürgen Jaskiewitz.

Frings, Theodor (1956): Sprache und Geschichte. Band I und II. Halle/Saale, Niemeyer.

Krueger, Joaquin; Graf de Krueger, Erna (1993): Hohenau. De la selva a la floreciente Colonia.

Obligado. Editor gráficolldeSilvero.

Müller, Horst M. (Hrsg.) (2002): ArbeitsbuchLinguistik. Paderborn, Schöningh.

Revista Distrital Obligado. Capital de los lácteos. Feliz $96^{\circ}$ aniversario. Obligado.

\section{DigitaleMedien}

Demokratiegeschichte. URL: http://www.demokratiegeschichte.eu (20.10.2011)

Deutsche Welle. URL: http://www.dw-world.de/ (15.09.2011)

Digitaler Wenker-Atlas (DiWA). URL: http://www.3.diwa.info/titel.aspx (5.10. 2011)

Duden. URL: http://www.duden.de/. (20.10.2011)

Hunsrückisch. Definition und Beispiele. URL:

http://www.linguateca.pt/GikiCLEF/GIRA/pool/GikiCLEF2009DocumentPool/de/h/u/n/Hunsr\% C3\%BCckisch.xml (27.10.2011)

Inmigración alemana en Brasil.

URL:http://es.wikipedia.org/wiki/Inmigraci\%C3\%B3n_alemana_en_Brasil (20.09.2011)

Instituto Brasileiro de Geografia e Estatística. URL:

http://www.ibge.gov.br/ibgeteen/datas/imigrante/brasil_e_os_imigrantes.html (24.09.2011)

Rheinische Geschichte. URL: http://www.rheinische-geschichte.lvr.de/ (15.09.2011)

Universidade Federal do Rio Grande do Sul. URL: http://www.ufrgs.br/projalma (20.10.2011) 